\title{
A convex relaxation approach to Set-membership identification of LPV systems
}

\author{
Vito Cerone $^{\mathrm{a}}$, Dario Piga ${ }^{\mathrm{b}}$, Diego Regruto ${ }^{\mathrm{a}}$ \\ ${ }^{a}$ Dipartimento di Automatica e Informatica, Politecnico di Torino, Corso Duca degli Abruzzi 24, 10129 Torino, Italy. \\ ${ }^{\mathrm{b}}$ Control Systems Group, Department of Electrical Engineering, Eindhoven University of Technology, P.O. Box 513, 5600 \\ MB, Eindhoven, The Netherlands.
}

\begin{abstract}
Identification of linear parameter varying models is considered in the paper, under the assumption that both the output and the scheduling parameter measurements are affected by bounded noise. First, the problem of computing parameter uncertainty intervals is formulated in terms of nonconvex optimization. Then, on the basis of the analysis of the regressor structure, we present an ad hoc convex relaxation scheme to compute parameter bounds by means of semidefinite optimization.
\end{abstract}

Key words: Bounded error identification, Convex relaxation, Linear Parameter Varying, LMI relaxation, Parameter bounds.

\section{Introduction}

Linear parameter varying (LPV) models can be defined, roughly speaking, as linear systems where either the matrices of the state equations or the coefficients of the difference equation relating the input and the output signals depend on one or more time varying parameters, whose real-time measurements are assumed to be available. These models have received a considerable attention from the identification and control community in recent years and can now be considered as one of the most popular paradigm to derive mathematical description of nonlinear/time-varying phenomena. As to the identification of LPV models, a significant number of contributions can be found in the literature since the work by Nemani, Ravikanth and Bamieh [12] which seems to be the first paper addressing the problem. A good deal of different approaches have been proposed including prediction-error minimization for LPV models with multiple scheduling variables [9], least square algorithms [1], subspace identification $[19,6,10,18]$, separable least squares [13], algorithm based on orthonormal basis expansions [17], just to cite a few. A detailed overview of the available LPV modeling and identification ap-

\footnotetext{
^ Corresponding author V. Cerone. Tel. +39-(0)11-5647064 Fax +39-(0)11-5647099.

Email addresses: vito.cerone@polito.it (Vito Cerone), D.Piga@tue.nl (Dario Piga), diego.regruto@polito.it (Diego Regruto).
}

proaches can be found in the recent book [16] by Tóth.

In all the papers mentioned above, the measurement error is statistically described. An alternative to the stochastic description of measurement errors is the bounded-errors or set-membership characterization, where uncertainties are assumed to belong to a given set (see, e.g., [11]). In this context, all parameters belonging to the feasible parameter set (FPS), i.e. parameters consistent with measurements, error bounds and the assumed model structure, are feasible solutions to the identification problem. To the authors' best knowledge, only a couple of contributions address the identification of LPV models when measurement errors are supposed to be bounded. In particular, the problem of identification and model validation of LPV systems in the presence of bounded noise and a possible nonparametric part is considered in [15]. A solution is proposed recasting the problem in terms of checking the feasibility of a set of linear matrix inequalities. In [2] the authors consider the identification of discrete-time LPV models with finite impulse response structure and output measurements affected by bounded noise.

In this paper, a procedure for set-membership identification of discrete-time LPV models when both the output and the time-varying parameter measurements are affected by bounded noise is considered. Preliminary results on this problem are presented in [5] and successfully applied to the problem of deriving an LPV model of 
the vehicle lateral dynamics in [3]. A new convex relaxation approach is proposed in this paper to compute uncertainty intervals on the system parameters by means of semidefinite optimization. The obtained bounds are proven to be tighter than those obtained in [5].

The paper is organized as follows. Section 2 is devoted to the problem formulation. In Section 3 we show that computation of tight parameter bounds requires the solution to nonconvex optimization problems. The proposed identification procedure is described in Section 4, where the peculiar structure of the considered problem is exploited to derive an original ad-hoc relaxation approach. A simulation example is reported in Section 5 to show the improvement of the presented procedure in the evaluation of the uncertainty intervals with respect to the algorithm proposed in [5].

\section{Problem formulation}

Consider the SISO discrete-time LPV model described in terms of the linear difference equation

$$
\mathcal{A}\left(q^{-1}, \lambda_{t}\right) w_{t}=\mathcal{B}\left(q^{-1}, \lambda_{t}\right) u_{t},
$$

where $u_{t}$ and $w_{t}$ are the input and the output signals respectively, while $\lambda_{t}=\left[\lambda_{t_{1}} \lambda_{t_{2}} \ldots \lambda_{t_{\mu}}\right]^{\mathrm{T}}$ is a vector of time-varying parameters which, according to the LPV modeling and control literature (see, e.g., [14]) are assumed to be measurable. $\mathcal{A}(\cdot)$ and $\mathcal{B}(\cdot)$ are polynomials in the backward shift operator $q^{-1}\left(q^{-1} w_{t}=w_{t-1}\right)$,

$$
\begin{gathered}
\mathcal{A}\left(q^{-1}, \lambda_{t}\right)=1+a_{1}\left(\lambda_{t}\right) q^{-1}+\ldots+a_{n a}\left(\lambda_{t}\right) q^{-n a}, \\
\mathcal{B}\left(q^{-1}, \lambda_{t}\right)=b_{0}\left(\lambda_{t}\right)+b_{1}\left(\lambda_{t}\right) q^{-1}+\ldots+b_{n b}\left(\lambda_{t}\right) q^{-n b},
\end{gathered}
$$

where $n a \geq n b$ and the coefficients $a_{i}$ and $b_{j}$ are assumed to be nonlinear memoryless mappings of parameters $\lambda_{t}$ described by

$$
\begin{aligned}
& a_{i}\left(\lambda_{t}\right)=\sum_{k=0}^{n_{i}} a_{i, k} \phi_{i, k}\left(\lambda_{t}\right), \\
& b_{j}\left(\lambda_{t}\right)=\sum_{h=0}^{m_{j}} b_{j, h} \psi_{j, h}\left(\lambda_{t}\right),
\end{aligned}
$$

where $\phi_{i, k}(\cdot)$ and $\psi_{j, h}(\cdot)$ are known nonlinear basis functions. In this work we assume that $\phi_{i, k}(\cdot)$ and $\psi_{j, h}(\cdot)$ belong to the canonical polynomial basis in the parameters $\lambda_{t}$, and we denote as $d_{\phi_{i, k}}$ and $d_{\psi_{j, h}}$ the degree of $\phi_{i, k}(\cdot)$ and $\psi_{j, h}(\cdot)$, respectively. Let $y_{t}$ and $z_{t}$ be the noise-corrupted measurements of $w_{t}$ and $\lambda_{t}$ respectively, i.e.

$$
\begin{aligned}
& y_{t}=w_{t}+\eta_{t}, \\
& z_{t}=\lambda_{t}+\varepsilon_{t},
\end{aligned}
$$

where $\varepsilon_{t}=\left[\varepsilon_{t_{1}} \varepsilon_{t_{2}} \ldots \varepsilon_{t_{\mu}}\right]^{\mathrm{T}}$. Measurement uncertainties $\eta_{t}$ and $\varepsilon_{t_{s}}$ are known to range within given bounds $\Delta \eta_{t}$ and $\Delta \varepsilon_{s_{t}}$, more precisely

$$
\left|\eta_{t}\right| \leq \Delta \eta_{t}
$$

and

$$
\varepsilon_{t} \in \mathcal{E}_{t}=\left\{\varepsilon_{t} \in \mathbb{R}^{\mu}:\left|\varepsilon_{t_{s}}\right| \leq \Delta \varepsilon_{t_{s}}, s=1,2, \ldots, \mu\right\} .
$$

The unknown parameter vector $\theta \in \mathbb{R}^{n_{\theta}}$ to be estimated is defined as

$$
\begin{aligned}
\theta^{\mathrm{T}}= & {\left[a_{1,0} \ldots a_{1, n_{1}} \ldots a_{n a, 0} \ldots a_{n a, n_{n a}}\right.} \\
& \left.b_{0,0} \ldots b_{0, m_{1}} \ldots b_{n b, 1} \ldots b_{n b, m_{n b}}\right]
\end{aligned}
$$

where $n_{\theta}=\sum_{i=1}^{n a} n_{i}+\sum_{j=0}^{n b} m_{j}$. The problem of deriving uncertainty intervals on the parameters $\theta$ is addressed in this paper. For the sake of simplicity and without loss of generality, in the rest of the paper we only consider the case of a scalar scheduling variable $\lambda_{t}$, that is $\lambda_{t} \in \mathbb{R}$. In order to make the paper easier to follow we introduce here a simple example that will be revisited in order to clarify the key technical arguments and formulas which are presented in the rest of the paper.

\section{Example 1}

Consider the problem of deriving uncertainty intervals for the SISO discrete-time LPV model described by

$$
w_{t}=-\left(a_{1,0}+a_{1,1} \lambda_{t}\right) w_{t-1}+b_{1,1} u_{t}
$$

using a set of $N=3$ measurements of the input, output and scheduling parameter, which are assumed to be affected by bounded noise according to equations (6)-(9).

\section{Evaluation of tight parameter bounds}

The set $\mathcal{D}$ of all the LPV system parameters $\theta$ and the noise samples $\varepsilon_{t}$ and $\eta_{t}$ consistent with the measurement data sequence, the assumed model structure and the error bounds is described by equations (1) - (9), i.e.

$$
\begin{gathered}
\mathcal{D}=\left\{(\theta, \eta, \varepsilon) \in \mathbb{R}^{n_{\theta}+N+(N-n a)}: \mathcal{A}\left(q^{-1}, z_{t}-\varepsilon_{t}\right)\left[y_{t}-\eta_{t}\right]\right. \\
=\mathcal{B}\left(q^{-1}, z_{t}-\varepsilon_{t}\right) u_{t},\left|\varepsilon_{t}\right| \leq \Delta \varepsilon_{t}, \quad\left|\eta_{r}\right| \leq \Delta \eta_{r} \\
t=n a+1, \ldots, N ; r=1, \ldots, N\}
\end{gathered}
$$

with $\eta=\left[\eta_{1}, \ldots, \eta_{N}\right]^{\mathrm{T}}$ and $\varepsilon=\left[\varepsilon_{n a+1}, \ldots, \varepsilon_{N}\right]^{\mathrm{T}}$. Therefore, for $j=1, \ldots, n_{\theta}$, tight bounds on the parameter $\theta_{j}$ can be computed by solving the optimization problems

$$
\underline{\theta}_{j}=\min _{(\theta, \eta, \varepsilon) \in \mathcal{D}} \theta_{j}, \quad \bar{\theta}_{j}=\max _{(\theta, \eta, \varepsilon) \in \mathcal{D}} \theta_{j}
$$

Parameter uncertainty intervals on $\theta_{j}$ are defined as $P U I_{j}=\left[\underline{\theta}_{j} ; \bar{\theta}_{j}\right]$.

\section{Example 2}

The set $\mathcal{D}$ for the identification problem introduced in 
Example 1 is given by:

$$
\begin{aligned}
\mathcal{D}=\{ & \left(a_{1,0}, a_{1,1}, b_{1,1}, \eta_{1}, \eta_{2}, \eta_{3}, \varepsilon_{2}, \varepsilon_{3}\right) \in \mathbb{R}^{8}: \\
& {\left[1+a_{1,0} q^{-1}+a_{1,1}\left(z_{t}-\varepsilon_{t}\right) q^{-1}\right]\left[y_{t}-\eta_{t}\right] } \\
& =b_{1,1} u_{t},\left|\varepsilon_{t}\right| \leq \Delta \varepsilon_{t}, \quad\left|\eta_{r}\right| \leq \Delta \eta_{r}, \\
& t=2,3 ; r=1,2,3\}
\end{aligned}
$$

Because of the polynomial constraints $\mathcal{A}\left(q^{-1}, z_{t}-\right.$ $\left.\varepsilon_{t}\right)\left[y_{t}-\eta_{t}\right]=\mathcal{B}\left(q^{-1}, z_{t}-\varepsilon_{t}\right) u_{t}$ defining the FPS $\mathcal{D}$, problems (13) belong to the class of semialgebraic optimization problems (see, e.g., [8] for details) which are, in general, nonconvex. Therefore, standard nonlinear optimization tools (gradient method, Newton method, etc.) can not be used because they can trap in local minima/maxima. As a consequence, the $P U I_{j}$ obtained by using these tools is not guaranteed to contain the true unknown parameter $\theta_{j}$, which is a key requirement of any set-membership identification method. A possible solution to overcome such a problem is to relax identification problems (13) into convex optimization problems in order to numerically compute lower bounds of $\underline{\theta}_{j}$ as well as upper bounds of $\bar{\theta}_{j}$. Optimization problems (13) enjoy an inherent structured sparsity due to the fact that, roughly speaking, the constraints defining $\mathcal{D}$ can be separated into a number of subsets of constraints, each one involving only a specific subset of optimization variables. More specifically, the constraints appearing in (13) can be shown to satisfy the so-called running intersection property (see, e.g., [8] for details) and, therefore, approximate solutions of $\underline{\theta}_{j}$ and $\bar{\theta}_{j}$ can be computed through a direct implementation of the sparse LMI relaxation proposed in [20,7]. Unfortunately, due to high memory usage, the relaxation order $\delta$ is supposed to be quite low in order to implement such an identification procedure in a commercial workstation (see [8] for details on the concept of relaxation order). Roughly, $\delta$ should be not greater than 2 when the number of parameters $\theta$ is 6 and the number $N$ of measurements is 30. In the authors' experience, relaxation of problems (13) through the direct implementation of the sparse LMI relaxation does not provide, in general, satisfactory bounds for a relaxation order $\delta=2$. The main reason is that, in the definition of the set $\mathcal{D}$, the parameters $\theta$ to be estimated are involved in polynomial constraints of degree greater or equal than 3. This means, in practice, that a relaxation order greater than 2 should be used in order to obtain a good approximation of $\underline{\theta}_{j}$ and $\bar{\theta}_{j}$. A tractable computational method for evaluating parameter bounds of LPV systems in the set-membership context is proposed in [5] where a linear programming relaxation of the nonconvex problems (13) is obtained by treating the successive occurrences of the uncertain variables appearing in the constraints defining (12) as independent variables. Although the relaxation method proposed in [5], called static approach, was shown to provide satisfactory results in general and was also successfully applied to an automotive real world problem in [3], it could lead, in some cases, to parameter bounds characterized by a significant amount of conservativeness due to the fact that the relaxation is obtained by neglecting the dynamic dependence across the different constraints defining $\mathcal{D}$. In this work we propose an alternative method to compute guaranteed bounds on the parameters $\theta$. Such a method provides parameter bounds significantly tighter than the ones obtained in [5] by exploiting an ad-hoc relaxation approach which partially retains the correlation between successive occurrences of the uncertain variables appearing in the constraints defining $\mathcal{D}$. For that reason, we call such a novel approach partial-dynamic LPV relaxation.

\section{Partial-dynamic LPV relaxation}

In this section we present a new technique to relax (13) into convex optimization problems. For the sake of clarity, a general overview of the proposed method is first presented in Section 4.1. Then, detailed technical results are provided in Section 4.2.

\subsection{Overview of the relaxation procedure}

Let us rewrite the FPS $\mathcal{D}$ defined by (12) in the matrix form

$$
\begin{gathered}
\mathcal{D}=\left\{(\theta, \eta, \varepsilon) \in \mathbb{R}^{n_{\theta}+2 N-n a}: A\left[\begin{array}{l}
\theta \\
1
\end{array}\right]=0, \quad\left|\varepsilon_{t}\right| \leq \Delta \varepsilon_{t},\right. \\
\left.\left|\eta_{r}\right| \leq \Delta \eta_{r}, \quad t=n a+1, \ldots, N ; r=1, \ldots, N\right\}
\end{gathered}
$$

where $A \in \mathbb{R}^{N-n a, n_{\theta}+1}$ and the $(t-n a)$-th row $A_{t-n a}$ of $A$ is

$$
\begin{aligned}
A_{t-n a}= & {\left[-\left(y_{t+n a}-\eta_{t+n a}\right) \phi_{1,0}\left(z_{t+n a+1}-\varepsilon_{t+n a+1}\right), \ldots,\right.} \\
& -\left(y_{t}-\eta_{t}\right) \phi_{n a, n_{n a}}\left(z_{t+n a+1}-\varepsilon_{t+n a+1}\right), \\
& u_{t+n a+1} \psi_{0,0}\left(z_{t+n a+1}-\varepsilon_{t+n a+1}\right), \ldots, \\
& u_{t+n a-n b} \psi_{n b, m_{n b}}\left(z_{t+n a+1}-\varepsilon_{t+n a+1}\right), \\
& \left.-y_{t+n a+1}+\eta_{t+n a+1}\right] .
\end{aligned}
$$

\section{Example 3}

With reference to the identification problem of Example 1 , the constraint in (14) corresponding to $t=2$ can be rewritten in the form:

$$
A_{1}\left[\begin{array}{c}
a_{1,0} \\
a_{1,1} \\
b_{1,1} \\
1
\end{array}\right]=0
$$


where

$$
A_{1}=-\left[\begin{array}{lll}
\left(y_{1}-\eta_{1}\right) & \left(z_{2}-\varepsilon_{2}\right)\left(y_{1}-\eta_{1}\right)-u_{2}\left(y_{2}-\eta_{2}\right)
\end{array}\right]
$$

The constraints corresponding to the other values of $t$ can be rewritten equivalently.

Note that the rows of the matrix $A$ are correlated with each other since the noise variable $\eta_{t}$ appears in all rows $A_{i}$, with $i=t-n a, t-n a+1, \ldots, t$. Besides, also the columns of the matrix $A$ are not independent of each other, since they are correlated by the noise variable affecting the scheduling parameter. The main idea of the partial-dynamic LPV relaxation can be summarized in the following steps:

(i) First, consider the rows of the matrix $A$ independent with each other, keeping the correlation between the columns. This leads to the construction of an outerbound $\mathcal{D}^{r}$ of the original feasible set $\mathcal{D}$.

(ii) Then, consider the columns of the matrix $A$ independent with each other, keeping the correlation between the rows. This leads to the construction of an outer-bound $\mathcal{D}^{c}$ of $\mathcal{D}$.

(iii) Define the relaxed feasible parameter set $\mathcal{D}^{r c}$ as $\mathcal{D}^{r c}=\mathcal{D}^{r} \cap \mathcal{D}^{c}$ and, for every $j=1, \ldots, n_{\theta}$, compute the minimum and the maximum value of the parameter $\theta_{j}$ over the feasible set $\mathcal{D}^{r c}$.

\subsection{Technical results}

\section{Result 1 Construction of the set $\mathcal{D}^{r}$}

Let us define the set $\mathcal{D}^{r}$ as

$$
\begin{aligned}
\mathcal{D}^{r}=\{ & (\theta, \eta, \varepsilon) \in \mathbb{R}^{n_{\theta}+2 N-n a}: \\
& \bar{A}_{t}^{r} \theta \geq y_{t}-\Delta \eta_{t}, \quad \underline{A}_{t}^{r} \theta \leq y_{t}+\Delta \eta_{t}, \\
& \left.\left|\varepsilon_{t}\right| \leq \Delta \varepsilon_{t}, \quad t=n a+1, \ldots, N\right\}
\end{aligned}
$$

where row vectors $\bar{A}_{t}^{r}$ and $\underline{A}_{t}^{r}$ are defined, respectively, as

$$
\begin{array}{r}
\bar{A}_{t}^{r}=\left[\left(-y_{t-1}+\Delta \eta_{t-1} \operatorname{sgn}\left(\phi_{1,0}\left(z_{t}-\varepsilon_{t}\right)\right) \operatorname{sgn}\left(a_{1,0}\right)\right) \phi_{1,0}, \ldots,\right. \\
\left(-y_{t-n a}+\Delta \eta_{t} \operatorname{sgn}\left(\phi_{n a, n_{n a}}\left(z_{t}-\varepsilon_{t}\right)\right) \operatorname{sgn}\left(a_{n a, n_{n a}}\right)\right) \phi_{n a, n_{n a}}, \\
\left.u_{t} \psi_{0,0}\left(z_{t}-\varepsilon_{t}\right), \ldots, u_{t-n b} \psi_{n b, m_{n b}}\left(z_{t}-\varepsilon_{t}\right)\right] .
\end{array}
$$

and

$$
\begin{array}{r}
\underline{A}_{t}^{r}=\left[\left(-y_{t-1}-\Delta \eta_{t-1} \operatorname{sgn}\left(\phi_{1,0}\left(z_{t}-\varepsilon_{t}\right)\right) \operatorname{sgn}\left(a_{1,0}\right)\right) \phi_{1,0}, \ldots,\right. \\
\left(-y_{t-n a}-\Delta \eta_{t} \operatorname{sgn}\left(\phi_{n a, n_{n a}}\left(z_{t}-\varepsilon_{t}\right)\right) \operatorname{sgn}\left(a_{n a, n_{n a}}\right)\right) \phi_{n a, n_{n a}}, \\
\left.u_{t} \psi_{0,0}\left(z_{t}-\varepsilon_{t}\right), \ldots, u_{t-n b} \psi_{n b, m_{n b}}\left(z_{t}-\varepsilon_{t}\right)\right] .
\end{array}
$$

Then, the set $\mathcal{D}^{r}$ is an outer-approximation of the feasible parameter set $\mathcal{D}$, i.e. $\mathcal{D}^{r} \supseteq \mathcal{D}$.
Proof Let us rewrite the constraint $\mathcal{A}\left(q^{-1}, z_{t}-\right.$ $\left.\varepsilon_{t}\right)\left[y_{t}-\eta_{t}\right]=\mathcal{B}\left(q^{-1}, z_{t}-\varepsilon_{t}\right) u_{t}$ defining $\mathcal{D}$ in (15) as

$$
\begin{aligned}
& \sum_{i=1}^{n a} \sum_{k=0}^{n_{i}}-a_{i, k} \phi_{i, k}\left(z_{t}-\varepsilon_{t}\right) y_{t-i}+\sum_{j=0}^{n b} \sum_{h=0}^{m_{j}} b_{j, h} \psi_{j, h}\left(z_{t}-\varepsilon_{t}\right) u_{t-j} \\
& -y_{t}=-\sum_{i=1}^{n a} \sum_{k=0}^{n_{i}} a_{i, k} \phi_{i, k}\left(z_{t}-\varepsilon_{t}\right) \eta_{t-i}-\eta_{t} .
\end{aligned}
$$

By taking the absolute value of both sides of eq. (22), from the triangle inequality and the condition $\left|\eta_{t}\right| \leq$ $\Delta \eta_{t}$, the following conditions hold:

$$
\begin{aligned}
& \left|\sum_{i=1}^{n a} \sum_{k=0}^{n_{i}}-a_{i, k} \phi_{i, k}\left(z_{t}-\varepsilon_{t}\right) y_{t-i}+\sum_{j=0}^{n b} \sum_{h=0}^{m_{j}} b_{j, h} \psi_{j, h}\left(z_{t}-\varepsilon_{t}\right) u_{t-j}-y_{t}\right| \\
& \leq \sum_{i=1}^{n a} \sum_{k=0}^{n_{i}}\left|a_{i, k}\right|\left|\phi_{i, k}\left(z_{t}-\varepsilon_{t}\right)\right|\left|\eta_{t-i}\right|+\left|\eta_{t}\right| \\
& \leq \sum_{i=1}^{n a} \sum_{k=0}^{n_{i}} \operatorname{sgn}\left(a_{i, k}\right) \operatorname{sgn}\left(\phi_{i, k}\left(z_{t}-\varepsilon_{t}\right)\right) a_{i, k} \phi_{i, k}\left(z_{t}-\varepsilon_{t}\right) \Delta \eta_{t-i} \\
& +\Delta \eta_{t} .
\end{aligned}
$$

Condition (23) can be written in the compact form

$$
\bar{A}_{t}^{r} \theta \geq y_{t}-\Delta \eta_{t} ; \quad \underline{A}_{t}^{r} \theta \leq y_{t}+\Delta \eta_{t}
$$

Therefore, when constraints $\mathcal{A}\left(q^{-1}, z_{t}-\varepsilon_{t}\right)\left[y_{t}-\eta_{t}\right]=$ $\mathcal{B}\left(q^{-1}, z_{t}-\varepsilon_{t}\right) u_{t}$ and $\left|\eta_{r}\right| \leq \Delta \eta_{r}$ (with $t=n a+1, \ldots, N$ and $r=1, \ldots, N)$ defining $\mathcal{D}$ are satisfied, also the constraints describing $\mathcal{D}^{r}$ are satisfied for all $t=1, \ldots, N-n a$. Thus, $\mathcal{D}^{r}$ is an outer-approximation of $\mathcal{D}$.

\section{Example 4}

With reference to the identification problem of Example 1 , let us rewrite the constraint in (14) corresponding to $t=2$ as follows:

$$
\begin{aligned}
& -y_{1} a_{1,0}-a_{1,1} y_{1}\left(z_{2}-\varepsilon_{2}\right)+b_{1,1} u_{2}-y_{2}= \\
& -\eta_{1} a_{1,0}-\eta_{1} a_{1,1}\left(z_{2}-\varepsilon_{2}\right)-\eta_{2}
\end{aligned}
$$

By taking the absolute value of both sides of eq. (25), from the triangle inequality and the condition $\left|\eta_{t}\right| \leq \Delta \eta_{t}$ one gets

$$
\begin{aligned}
& \left|-y_{1} a_{1,0}-a_{1,1} y_{1}\left(z_{2}-\varepsilon_{2}\right)+b_{1,1} u_{2}-y_{2}\right| \\
& \leq\left|\eta_{1}\right|\left|a_{1,0}\right|+\left|\eta_{1}\right|\left|a_{1,1}\right|\left|\left(z_{2}-\varepsilon_{2}\right)\right|+\left|\eta_{2}\right| \\
& \leq\left|\eta_{1}\right| \operatorname{sgn}\left(a_{1,0}\right) a_{1,0}+ \\
& +\left|\eta_{1}\right| \operatorname{sgn}\left(a_{1,1}\right) a_{1,1} \operatorname{sgn}\left(z_{2}-\varepsilon_{2}\right)\left(z_{2}-\varepsilon_{2}\right)+\left|\eta_{2}\right| \\
& \leq \Delta \eta_{1} \operatorname{sgn}\left(a_{1,0}\right) a_{1,0} \\
& +\Delta \eta_{1} \operatorname{sgn}\left(a_{1,1}\right) a_{1,1} \operatorname{sgn}\left(z_{2}-\varepsilon_{2}\right)\left(z_{2}-\varepsilon_{2}\right)+\Delta \eta_{2} .
\end{aligned}
$$


Condition (26) is equivalent to the following pair of inequalities:

$$
\bar{A}_{2}^{r} \theta \geq y_{2}-\Delta \eta_{2}, \quad \underline{A}_{2}^{r} \theta \leq y_{2}+\Delta \eta_{2}
$$

where

$$
\begin{aligned}
& \bar{A}_{2}^{r}=\left[-y_{1}+\Delta \eta_{1} \operatorname{sgn}\left(a_{1,1}\right) u_{2}\right] \\
& \underline{A}_{2}^{r}=\left[-y_{1}-\Delta \eta_{1} \operatorname{sgn}\left(a_{1,1}\right) u_{2}\right] .
\end{aligned}
$$

In order to construct the outer-approximation $\mathcal{D}^{c}$ of $\mathcal{D}$ we first provide the following definitions:

$$
\begin{gathered}
\underline{\phi}_{i, k}^{t}=\min _{\left|\varepsilon_{t}\right| \leq \Delta \varepsilon_{t}} \phi_{i, k}^{t}\left(z_{t}-\varepsilon_{t}\right), \quad \bar{\phi}_{i, k}^{t}=\max _{\left|\varepsilon_{t}\right| \leq \Delta \varepsilon_{t}} \phi_{i, k}^{t}\left(z_{t}-\varepsilon_{t}\right), \\
\underline{\gamma}_{j, h}^{t}=\min _{\left|\varepsilon_{t}\right| \leq \Delta \varepsilon_{t}} \gamma_{j, h}^{t}, \quad \bar{\gamma}_{j, h}^{t}=\max _{\left|\varepsilon_{t}\right| \leq \Delta \varepsilon_{t}} \gamma_{j, h}^{t},
\end{gathered}
$$

and

$$
\begin{aligned}
& c\left(\phi_{i, k}^{t}\right)=\frac{\bar{\phi}_{i, k}^{t}+\underline{\phi}_{i, k}^{t}}{2}, \quad \Delta \phi_{i, k}^{t}=\frac{\bar{\phi}_{i, k}^{t}-\underline{\phi}_{i, k}^{t}}{2}, \\
& c\left(\gamma_{j, h}^{t}\right)=\frac{\bar{\gamma}_{j, h}^{t}+\underline{\gamma}_{j, h}^{t}}{2}, \quad \Delta \gamma_{j, h}^{t}=\frac{\bar{\gamma}_{j, h}^{t}-\underline{\gamma}_{j, h}^{t}}{2} .
\end{aligned}
$$

Remark 1 Since $\phi_{i, k}^{t}(\cdot)$ and $\psi_{j, h}^{t}(\cdot)$ are continuous functions, the Weierstrass theorem guarantees that $\phi_{i, k}^{t}(\cdot)$ and $\gamma_{j, h}^{t}(\cdot)$ achieve their global minimum and maximum on the closed interval $\left|\varepsilon_{t}\right| \leq \Delta \varepsilon_{t}$. As is well known, such a global minimum and maximum must either be stationary points or lie on the boundary of the interval $\left|\varepsilon_{t}\right| \leq \Delta \varepsilon_{t}$ and their computation is straightforward as $\phi_{i, k}^{t}(\cdot)$ and $\psi_{j, h}^{t}(\cdot)$ are polynomial functions.

\section{Result 2 Construction of the set $\mathcal{D}^{c}$}

Let us define the set $\mathcal{D}^{c}$ as

$$
\begin{aligned}
\mathcal{D}^{c}=\{ & (\theta, \eta, \varepsilon) \in \mathbb{R}^{n_{\theta}+2 N-n a}: \\
& \left(A_{t}^{c}+\Delta A_{t}^{c}\right) \theta \geq y_{t}-\eta_{t}, \quad\left(A_{t}^{c}-\Delta A_{t}^{c}\right) \theta \leq y_{t}-\eta_{t}, \\
& \left.\left|\eta_{t}\right| \leq \Delta \eta_{t}, \quad t=n a+1, \ldots, N\right\},
\end{aligned}
$$

where row vectors $A_{t}^{c}$ and $\Delta A_{t}^{c}$ are defined as

$$
\begin{aligned}
A_{t}^{c} & =\left[-\left(y_{t-1}-\eta_{t-1}\right) c\left(\phi_{1,0}^{t}\right), \ldots,\right. \\
& \left.-\left(y_{t-n a}-\eta_{t-n a}\right) c\left(\phi_{n a, n_{n a}}^{t}\right), c\left(\gamma_{0,0}^{t}\right), \ldots, c\left(\gamma_{n b, m_{n b}}^{t}\right)\right],
\end{aligned}
$$

$\Delta A_{t}^{c}=\left[\left(y_{t-1}-\eta_{t-1}\right) \Delta \phi_{1,0}^{t} \operatorname{sgn}\left(y_{t-1}-\eta_{t-1}\right) \operatorname{sgn}\left(a_{1,0}\right), \ldots\right.$ $\left(y_{t-n a}-\eta_{t-n a}\right) \Delta \phi_{n a, n_{n a}}^{t} \operatorname{sgn}\left(y_{t-n a}-\eta_{t-n a}\right) \operatorname{sgn}\left(a_{n a, n_{n a}}\right)$, $\left.\Delta \gamma_{0,0}^{t} \operatorname{sgn}\left(b_{0,0}\right), \ldots, \Delta \gamma_{n b, m_{n b}}^{t} \operatorname{sgn}\left(b_{n b, m_{n b}}\right)\right]$.
Then, the set $\mathcal{D}^{c}$ is an outer-approximation of the FPS $\mathcal{D}$, i.e. $\mathcal{D}^{c} \supseteq \mathcal{D}$.

Proof Let us write $\phi_{i, k}^{t}$ (respectively $\gamma_{j, h}^{t}$ ) in terms of its central value $c\left(\phi_{i, k}^{t}\right)$ (respectively $\left.c\left(\gamma_{j, h}^{t}\right)\right)$ and its perturbation $\delta \phi_{i, k}^{t}$ (respectively $\left.\delta \gamma_{j, h}^{t}\right)$, that is

$$
\phi_{i, k}^{t}=c\left(\phi_{i, k}^{t}\right)+\delta \phi_{i, k}^{t}, \quad \gamma_{j, h}^{t}=c\left(\gamma_{j, h}^{t}\right)+\delta \gamma_{j, h}^{t} .
$$

Indeed,

$$
\left|\delta \phi_{i, k}^{t}\right| \leq \Delta \phi_{i, k}^{t}, \quad\left|\delta \gamma_{j, h}^{t}\right| \leq \Delta \gamma_{j, h}^{t}
$$

Then, let us rewrite the constraint $\mathcal{A}\left(q^{-1}, z_{t}-\right.$ $\left.\varepsilon_{t}\right)\left[y_{t}-\eta_{t}\right]=\mathcal{B}\left(q^{-1}, z_{t}-\varepsilon_{t}\right) u_{t}$ defining $\mathcal{D}$ in (15) as

$$
\begin{aligned}
& \sum_{i=1}^{n a} \sum_{k=0}^{n_{i}}-a_{i, k}\left(y_{t-i}-\eta_{t-i}\right) c\left(\phi_{i, k}^{t}\right)+ \\
& \sum_{j=0}^{n b} \sum_{h=0}^{m_{j}} b_{j, h} c\left(\gamma_{j, h}^{t}\right)-y_{t}+\eta_{t}= \\
& =\sum_{i=1}^{n a} \sum_{k=0}^{n_{i}} a_{i, k}\left(y_{t-i}-\eta_{t-i}\right) \delta \phi_{i, k}^{t}-\sum_{j=0}^{n b} \sum_{h=0}^{m_{j}} b_{j, h} \delta \gamma_{j, h}^{t} .
\end{aligned}
$$

Through algebraic manipulations similar to the ones used in the proof of Result 1 and since $\left|\delta \phi_{i, k}^{t}\right| \leq \Delta \phi_{i, k}^{t}$ and $\left|\delta \gamma_{j, h}^{t}\right| \leq \Delta \gamma_{j, h}^{t}$, the following inequalities hold:

$\mid \sum_{i=1}^{n a} \sum_{k=0}^{n_{i}}-a_{i, k}\left(y_{t-i}-\eta_{t-i}\right) c\left(\phi_{i, k}^{t}\right)+$

$\sum_{j=0}^{n b} \sum_{h=0}^{m_{j}} b_{j, h} c\left(\gamma_{j, h}^{t}\right)-y_{t}+\eta_{t} \mid \leq$

$\sum_{i=1}^{n a} \sum_{k=0}^{n_{i}} a_{i, k}\left(y_{t-i}-\eta_{t-i}\right) \operatorname{sgn}\left(a_{i, k}\right) \operatorname{sgn}\left(y_{t-i}-\eta_{t-i}\right) \Delta \phi_{i, k}^{t}+$

$\sum_{j=0}^{n b} \sum_{h=0}^{m_{j}} b_{j, h} \operatorname{sgn}\left(b_{j, h}\right) \Delta \gamma_{j, h}^{t}$.

Conditions in (39) can be also written in the compact form

$$
\left(A_{t}^{c}+\Delta A_{t}^{c}\right) \theta \geq y_{t}-\eta_{t}, \quad\left(A_{t}^{c}-\Delta A_{t}^{c}\right) \theta \leq y_{t}-\eta_{t} .
$$

Thus, when the constraints $\mathcal{A}\left(q^{-1}, z_{t}-\varepsilon_{t}\right)\left[y_{t}-\eta_{t}\right]=$ $\mathcal{B}\left(q^{-1}, z_{t}-\varepsilon_{t}\right) u_{t}$ and $\left|\varepsilon_{t}\right| \leq \Delta \varepsilon_{t}($ with $t=n a+1, \ldots, N)$ defining $\mathcal{D}$ are satisfied, also the inequalities in (40), which in turn describe $\mathcal{D}^{c}$, are satisfied for all $t=n a+1, \ldots, N$. Then, the set $\mathcal{D}^{c}$ is an outerapproximation of the FPS $\mathcal{D}$. 
An outer-approximation of the FPS $\mathcal{D}$ tighter than both $\mathcal{D}^{r}$ and $\mathcal{D}^{c}$ can be then defined as the intersection of $\mathcal{D}^{r}$ and $\mathcal{D}^{c}$, i.e.

$$
\mathcal{D}^{r c}=\mathcal{D}^{r} \cap \mathcal{D}^{c} .
$$

Then, bounds on the parameters $\theta_{j}$ can be computed by solving the optimization problems

$$
\underline{\theta}_{j}^{p d}=\min _{(\theta, \eta, \varepsilon) \in \mathcal{D}^{r c}} \theta_{j}, \quad \bar{\theta}_{j}^{p d}=\max _{(\theta, \eta, \varepsilon) \in \mathcal{D}^{r c}} \theta_{j},
$$

and the parameter uncertainty interval on $\theta_{j}$ obtained through the partial-dynamic LPV relaxation is then defined as $P U I_{j}^{p d}=\left[\underline{\theta}_{j}^{p d} ; \bar{\theta}_{j}^{p d}\right]$.

\section{Property 1 Accuracy improvement of $P U I_{j}^{p d}$ over $P U I_{j}^{s}$}

For all $j=1, \ldots, n_{\theta}$, the parameter uncertainty interval $P U I_{j}^{p d}$ is tighter than the interval $P U I_{j}^{s}$ (obtained through the static LPV relaxation proposed in [5] and briefly reviewed in Section 5), i.e. $P U I_{j}^{p d} \subseteq P U I_{j}^{s}$.

Proof The proof is based on the fact that both $\mathcal{D}^{r}$ and $\mathcal{D}^{c}$ are, by construction, subsets of $\mathcal{D}^{s}$, where $\mathcal{D}^{s}$ denotes the outer-approximation of the feasible parameter set (12) derived in [5]. The reader is referred to the technical report [4] for details.

\subsection{Computation of parameter bounds $\underline{\theta}_{j}^{p d}$ and $\bar{\theta}_{j}^{p d}$}

By exploiting the peculiar structure of the set $\mathcal{D}^{r c}$, we now show that parameter bounds $\underline{\theta}_{j}^{p d}$ and $\bar{\theta}_{j}^{p d}$ can be computed through the solution of a set of semialgebraic optimization problems.

\section{Property 2 Topological features of the feasible} set $\mathcal{D}^{r c}$

If the relative measurement errors on both the output $w_{t}$ and on the scheduling variable $\lambda_{t}$ is smaller than $100 \%$, i.e. $\left|\eta_{t}\right| \leq\left|w_{t}\right|$ and $\left|\varepsilon_{t}\right| \leq\left|\lambda_{t}\right|$, then the set $\mathcal{D}^{r c}$ is the union of at most $2^{n_{\theta}}$ sets $\overline{\mathcal{D}}_{i}^{r c}$ in $\mathbb{R}^{n_{\theta}+2 N-n a}$, i.e.

$$
\mathcal{D}^{r c}=\bigcup_{i=1}^{2^{n_{\theta}}} \mathcal{D}_{i}^{r c} .
$$

The set $\mathcal{D}_{i}^{r c}$ is the intersection of $\mathcal{D}^{r c}$ with the i-th orthant $\mathcal{O}_{i}$ of the parameter space $\mathbb{R}^{n_{\theta}}$, i.e.

$$
\mathcal{D}_{i}^{r c}=\mathcal{D}^{r c} \cap \mathcal{O}_{i}
$$

The orthant $\mathcal{O}_{i}$ is formally described as

$$
\mathcal{O}_{i}=\left\{\theta \in \mathbb{R}^{n_{\theta}}: \alpha_{i_{j}} \theta_{j} \geq 0, j=1, \ldots, n_{\theta}\right\}
$$

where $\alpha_{i} \in \Gamma$, being $\Gamma$ the set of all $n_{\theta}$-dimensional vectors with components equal to either +1 or -1 and $\alpha_{i_{j}}$ denotes the $j$-th component of $\alpha_{i}$.

Besides, each set $\mathcal{D}_{i}^{r c}$, if not empty, is a semialgebraic region in $\mathbb{R}^{n_{\theta}+2 N-n a}$ defined by polynomial inequalities of maximum degree $d_{\theta}^{r c}=\max \left\{1+\max _{i, k}\left\{d_{\phi_{i, k}}\right\}, 1+\right.$ $\left.\max _{j, h}\left\{d_{\psi_{j, h}}\right\}, 2\right\}$.

The reader is referred to the technical report [4] for a detailed proof of property 2 .

Remark 2 The assumption that the relative error on the measurements of $w_{t}$ and $\lambda_{t}$ is smaller than $100 \%$ is a sufficient condition to state that the sign of $y_{t}-\eta_{t}$ and $z_{t}-\varepsilon_{t}$ is known. If such an assumption is not satisfied, then the set $\mathcal{D}^{r c}$ is the union of at most $2^{n_{\theta}+2 N-n a}$ semialgebraic sets.

Thanks to Property 2, identification problems (42) can be decomposed into a collection of polynomial optimization problems. In fact, solving (42) is equivalent to compute

$$
\underline{\theta}_{j}^{p d}=\min _{l=1, \ldots, 2^{n_{\theta}}} \underline{\theta}_{j i}^{p d}, \quad \bar{\theta}_{j}^{p d}=\max _{l=1, \ldots, 2^{n_{\theta}}} \bar{\theta}_{j i}^{p d},
$$

where $\underline{\theta}_{j}^{p d}$ and $\bar{\theta}_{j}^{p d}$ are solutions to the following semialgebraic optimization problems:

$$
\underline{\theta}_{j i}^{p d}=\min _{(\theta, \eta, \varepsilon) \in \mathcal{D}_{i}^{r c}} \theta_{j}, \quad \bar{\theta}_{j i}^{p d}=\max _{(\theta, \eta, \varepsilon) \in \mathcal{D}_{i}^{r c}} \theta_{j} .
$$

Analysis of the structure of the constraints defining the sets $\mathcal{D}_{i}^{r c}$ reveals that they can be separated into a number of subsets of constraints, each one involving only a specific subset of the optimization variables (see [4] for details). Besides, the constraints appearing in (13) can be shown to satisfy the so-called running intersection property (see, e.g., [8] for details) and, therefore, approximate solutions to problems (47) can be computed through a direct implementation of the sparse LMI relaxation proposed in $[20,7]$.

For a given relaxation order $\delta$, let us define the $\delta$-relaxed uncertainty intervals obtained through the partialdynamic-LPV procedure as

$$
P U I_{j}^{p d, \delta}=\left[\underline{\theta}_{j}^{p d, \delta} ; \bar{\theta}_{j}^{p d, \delta}\right],
$$

where

$$
\underline{\theta}_{j}^{p d, \delta}=\min _{i=1, \ldots, 2^{n_{\theta}}} \underline{\theta}_{j i}^{p d, \delta}, \quad \bar{\theta}_{j}^{p d, \delta}=\max _{i=1, \ldots, 2^{n_{\theta}}} \bar{\theta}_{j i}^{p d, \delta} .
$$

Property 3 For all $j=1, \ldots, n_{\theta}$, the $\delta$-relaxed parameter uncertainty interval $P U I_{j}^{p d, \delta}$ satisfies the following properties. 


\section{P 3.1 Guaranteed relaxed uncertainty intervals} For any relaxation order $\delta \geq \underline{\delta}$, the $\delta$-relaxed parameter uncertainty interval $P U I_{j}^{p \bar{d}, \delta}$ is guaranteed to contain the true unknown parameter $\theta_{j}$ to be estimated, i.e. $\theta_{j} \in$ $P U I_{j}^{p d, \delta}$.

\section{P 3.2 Monotone convergence to intervals $P U I_{j}^{p d}$}

The $\delta$-relaxed parameter uncertainty interval $P U I_{j}^{p d, \delta}$ becomes tighter as the relaxation order $\delta$ increases, that is

$$
P U I_{j}^{p d, \delta+1} \subseteq P U I_{j}^{p d, \delta} .
$$

Furthermore, as the LMI relaxation order goes to infinity, the $\delta$-relaxed parameter uncertainty interval $P U I_{j}^{p d, \delta}$ converges to the interval $P U I_{j}^{p d}$, that is $\lim _{\delta \rightarrow \infty} \underline{\theta}_{j}^{p d, \delta}=\underline{\theta}_{j}^{p d}$ and $\lim _{\delta \rightarrow \infty} \bar{\theta}_{j}^{p d, \delta}=\bar{\theta}_{j}^{p d}$.

Properties P3.1 and P3.2 follow from the fact that the structures of the constraints of the optimization problem (47) satisfies the running intersection property (see [4] for details) and, therefore, results from [7] about the monotone convergence of the sparse relaxation approach proposed in [20] apply to the proposed partial-dynamic method.

\section{A simulation example}

In this section, the capabilities of the presented LPV identification scheme are demonstrated by means of a simulation example, where we propose a comparison between the proposed partial-dynamic method and the so-called static approach proposed in [5].

The true data-generating LPV system considered here is described by (1) with $\mathcal{A}\left(q^{-1}, \lambda_{t}\right)=1+$ $0.5 \lambda_{t} q^{-1}+\left(-0.3+0.5 \lambda_{t}^{2}\right) q^{-2}$ and $\mathcal{B}\left(q^{-1}, \lambda_{t}\right)=$ $0.3 q^{-1}+\left(1.2 \lambda_{t}+0.6 \lambda_{t}^{2}\right) q^{-2}$. Therefore, the true parameters vector is $\theta=\left[a_{1,1}, a_{2,0}, a_{2,2}, b_{1,0}, b_{2,1}, b_{2,2}\right]^{\mathrm{T}}=$ $[0.5,-0.3,0.5,0.3,1.2,0.6]^{\mathrm{T}}$ and the functions $\phi_{i, k}$ and $\psi_{j, h}$ in (4) and (5), which depend on the scheduling parameter $\lambda_{t}$, are $\phi_{1,1}=\lambda_{t}, \phi_{2,0}=1, \phi_{2,2}=\lambda_{t}^{2}$, $\psi_{1,0}=1, \psi_{2,1}=\lambda_{t}$ and $\psi_{2,2}=\lambda_{t}^{2}$. The input sequence $\left\{u_{t}\right\}$ is a random uniform distributed signal which takes values in the interval $[-1,1]$ and the scheduling parameter $\lambda_{t}$ is such that $\lambda_{t}=\cos (0.3 t)$. The output $w_{t}$ and the scheduling signal $\lambda_{t}$ are corrupted by random additive noises $\eta_{t}$ and $\varepsilon_{t}$, respectively, uniformly distributed in $\left[-\Delta \eta_{t},+\Delta \eta_{t}\right]$ and $\left[-\Delta \varepsilon_{t},+\Delta \varepsilon_{t}\right]$. The chosen error bounds $\Delta \eta_{t}$ and $\Delta \varepsilon_{t}$ are such that the signal to noise ratios on the output $S N R_{w}$ and on the scheduling signal $S N R_{\lambda}$, defined as:

$$
S N R_{w}=10 \log \left\{\frac{\sum_{t=1}^{N} w_{t}^{2}}{\sum_{t=1}^{N} \eta_{t}^{2}}\right\} ; S N R_{\lambda}=10 \log \left\{\frac{\sum_{t=1}^{N} \lambda_{t}^{2}}{\sum_{t=1}^{N} \varepsilon_{t}^{2}}\right\},
$$

are $23 \mathrm{db}$ and $24 \mathrm{db}$, respectively. The number of measurements $N$ used to compute the parameter bounds is 400. First, bounds on the parameters are evaluated through the static LPV approach proposed in [5]. The obtained relaxed bounds $\underline{\theta}_{j}^{s}, \bar{\theta}_{j}^{s}$, the central estimate $\theta_{j}^{c s}$ and the parameter uncertainty bounds $\Delta \theta_{j}^{s}$, defined as

$$
\theta_{j}^{c s}=\frac{\bar{\theta}_{j}^{s}+\underline{\theta}_{j}^{s}}{2}, \quad \Delta \theta_{j}^{s}=\frac{\bar{\theta}_{j}^{s}-\underline{\theta}_{j}^{s}}{2},
$$

are reported in Table 1 . Indeed, the width of the interval $P U I_{j}^{s}$ is $2 \Delta \theta_{j}^{s}$.

The elapsed time to compute a single parameter bound $\left(\underline{\theta}_{j}^{s}\right.$ or $\left.\bar{\theta}_{j}^{s}\right)$ by using the linprog function in Matlab, is between $0.71 \mathrm{~s}$ and $0.93 \mathrm{~s}$.

Then, parameter bounds are evaluated through the partial-dynamic LPV relaxation proposed in this paper. The obtained parameter bounds $\underline{\theta}_{j}^{p d, \delta}$ and $\bar{\theta}_{j}^{p d, \delta}$, computed for a relaxation order $\delta=2$, are reported in Table 2 together with the central estimate $\theta_{j}^{c p d, \delta}$ and the parameter uncertainty bounds $\Delta \theta_{j}^{p d, \delta}$ defined as

$$
\theta_{j}^{c p d, \delta}=\frac{\bar{\theta}_{j}^{p d, \delta}+\underline{\theta}_{j}^{p d, \delta}}{2}, \Delta \theta_{j}^{p d, \delta}=\frac{\bar{\theta}_{j}^{p d, \delta}-\underline{\theta}_{j}^{p d, \delta}}{2} .
$$

The CPU elapsed time taken by the SeDuMi solver to evaluate a single parameter bound $\left(\underline{\theta}_{j}^{p d, \delta}\right.$ or $\left.\bar{\theta}_{j}^{p d, \delta}\right)$ is between $330 \mathrm{~s}$ and $361 \mathrm{~s}$. Results in Tables 1 and 2 show that the true parameters are always included in the parameter uncertainty intervals, as expected. Furthermore, a comparison of such results shows that the partial-dynamic LPV relaxation provides parameter bounds tighter than the ones obtained through the method proposed in [5]. As a matter of fact, even if a low value of the relaxation order $\delta$ is used, for each parameter $\theta_{j}$, it results that $\frac{\Delta \theta_{j}^{s}}{\Delta \theta_{j}^{p d, \delta}} \geq 1.5$.

Table 1

Parameter central estimates $\left(\theta_{j}^{c s}\right)$, parameter bounds $\left(\underline{\theta}_{j}^{s}\right.$, $\bar{\theta}_{j}^{s}$ ) and parameter uncertainties $\Delta \theta_{j}^{s}$ obtained through the static LPV relaxation.

\begin{tabular}{cccccc}
\hline \multirow{2}{*}{ Parameter } & $\begin{array}{c}\text { True } \\
\text { Value }\end{array}$ & $\underline{\theta}_{j}^{s}$ & $\theta_{j}^{c s}$ & $\bar{\theta}_{j}^{s}$ & $\Delta \theta_{j}^{s}$ \\
\hline$a_{1,1}$ & 0.500 & 0.366 & 0.512 & 0.658 & 0.146 \\
$a_{2,0}$ & -0.300 & -0.348 & -0.281 & -0.214 & 0.067 \\
$a_{2,2}$ & 0.500 & 0.382 & 0.507 & 0.632 & 0.125 \\
$b_{1,0}$ & 0.300 & 0.206 & 0.342 & 0.478 & 0.136 \\
$b_{2,1}$ & 1.200 & 0.939 & 1.391 & 1.843 & 0.452 \\
$b_{2,2}$ & 0.600 & 0.298 & 0.574 & 0.850 & 0.276 \\
\hline
\end{tabular}


Table 2

Parameter central estimates $\left(\theta_{j}^{c p d, \delta}\right)$, parameter bounds $\left(\underline{\theta}_{j}^{p d, \delta}, \bar{\theta}_{j}^{p d, \delta}\right)$ and parameter uncertainties $\Delta \theta_{j}^{p d, \delta}$ obtained through the partial dynamic LPV relaxation for a relaxation order $\delta=2$.

\begin{tabular}{|c|c|c|c|c|c|}
\hline Parameter & $\begin{array}{l}\text { True } \\
\text { Value }\end{array}$ & $\underline{\theta}_{j}^{p d, \delta}$ & $\theta_{j}^{c p d, \delta}$ & $\bar{\theta}_{j}^{p d, \delta}$ & $\Delta \theta_{j}^{p d, \delta}$ \\
\hline$a_{1,1}$ & 0.500 & 0.410 & 0.507 & 0.604 & 0.097 \\
\hline$a_{2,0}$ & -0.300 & -0.340 & -0.295 & -0.250 & 0.045 \\
\hline$a_{2,2}$ & 0.500 & 0.418 & 0.493 & 0.568 & 0.075 \\
\hline$b_{1,0}$ & 0.300 & 0.224 & 0.311 & 0.398 & 0.087 \\
\hline$b_{2,1}$ & 1.200 & 1.026 & 1.243 & 1.460 & 0.217 \\
\hline$b_{2,2}$ & 0.600 & 0.453 & 0.618 & 0.783 & 0.165 \\
\hline
\end{tabular}

\section{Conclusions}

A new technique to evaluate parameter uncertainty intervals for LPV systems when both the output and the scheduling signal measurements are affected by bounded noise is presented in this paper. Parameter bounds evaluation is formulated in terms of nonconvex optimization problems. In order to reduce the computation complexity of the identification problem, the original feasible parameter set is approximated by the union of semialgebraic regions described by polynomial inequalities that involve only a small number of decision variables. Thanks to the structured sparsity of the identification problem, relaxation techniques based on linear matrix inequalities are exploited to compute parameter uncertainty intervals, which are guaranteed to contain the true parameters. The capability of the proposed identification technique to provide a less conservative estimate of parameter bounds with respect to the previously published results is shown both theoretically and by means of a numerical example.

\section{Acknowledgements}

This research was developed while Dr. D. Piga was a Ph.D student at the Politecnico di Torino.

\section{References}

[1] B. A. Bamieh and L. Giarré. Identification of linear parameter-varying models. International Journal of Robust and Nonlinear Control, 12(9):841-853, 2002.

[2] G. Belforte and P. Gay. Optimal worst case estimation for LPV-FIR models with bounded errors. Systems \& Control letters, 53:259-268, 2004.

[3] V. Cerone, D. Piga, and D. Regruto. Set-membership LPV model identification of vehicle lateral dynamics. Automatica, 47(8):1794-1799, 2011.
[4] V. Cerone, D. Piga, and D. Regruto. Ad-hoc relaxation techniques for bounded-error identification of LPV systems. Internal Report, DAUIN(2013/02/31cepire):available upon request, 2013.

[5] V. Cerone and D. Regruto. Set-membership identification of LPV models with uncertain measurements of the timevarying parameter. In Proc. of the 47th IEEE Conference on Decision and Control, pages 4491-4496, 2008.

[6] F. Felici, J. W. Van Wingerden, and M. Verhaegen. Subspace identification of MIMO LPV systems using a periodic scheduling sequence. Automatica, 43:1684-1697, 2007.

[7] J. B. Lasserre. Convergent semidefinite relaxations in polynomial optimization with sparsity. SIAM Journal on Optimization, 17(1):822-843, 2006.

[8] J.B. Lasserre. Moments, Positive Polynomials and Their Applications. Imperial College Press, Optimization series, Vol. 1, 2010.

[9] L. H. Lee and K. Poolla. Identification of linear parametervarying systems using nonlinear programming. ASME Journal of Dynami Systems, Measurement and Control, 121:71-78, 1999.

[10] P. Lopes dos Santos, J. A. Ramos, and J. L. Martins de Carvalho. Subspace identification of linear parameter-varying systems with innovation-type noise models driven by general inputs and a measurable white noise time-varying parameter vector. Int. J. of Systems Science, 39(9):897-911, 2008.

[11] M. Milanese, J. Norton, H. Piet-Lahanier, and E. Walter, editors. Bounding approaches to system identification. Plenum Press, New York, 1996.

[12] M. Nemani, R. Ravikanth, and B. A. Bamieh. Identification of linear parametrically varying sistems. In Proceedings of 34th Conference on Decision and Control, pages 2990-2995, 1995.

[13] F. Previdi and M. Lovera. Identification of non-linear parametrically varying models using separable least squares. International Journal of Control, 77(16):1382-1392, 2004.

[14] W. J. Rugh and J. S. Shamma. Research on gain scheduling. Automatica, 36(10):1401-1425, 2000.

[15] M. Sznaier and C. Mazzarro. An LMI approach to control-oriented identification and model (in)validation of LPV systems. IEEE Transactions on Automatic Control, 48(9):1619-1624, 2003.

[16] R. Tóth. Modeling and identification of linear parametervarying systems. Lecture Notes in Control and Information Sciences, 403. Springer Germany, 2010.

[17] R. Tóth, P. S. C. Heuberger, and P. M. J. Van den Hof. Asymptotically optimal orthonormal basis functions for LPV system identification. Automatica, 45(6):1359-1370, 2010.

[18] V. Verdult, M. Lovera, and M. Verhaegen. Identification of linear parameter-varying state-space models with application to helicopter rotor dynamics. International Journal of Control, 77(13):1149-1159, 2004.

[19] V. Verdult and M. Verhaegen. Subspace identification of multivariable linear parameter-varying systems. Automatica, 38:805-814, 2002.

[20] H. Waki, S. Kim, M. Kojima, and M. Muramatsu. Sums of squares and semidefinite programming relaxations for polynomial optimization problems with structured sparsity. SIAM Journal on Optimization, 17(1):218-242, 2006. 